\title{
Spindle Cell Oncocytoma
}

National Cancer Institute

\section{Source}

National Cancer Institute. Spindle Cell Oncocytoma. NCI Thesaurus. Code C94537.

A very rare, WHO grade I neoplasm of the posterior pituitary. It is characterized by the presence of spindle cells with eosinophilic, granular cytoplasm forming fascicles. Electron microscopic studies demonstrate the accumulation of intracytoplasmic mitochondria and lack of secretory granules. Immunohistochemical studies are negative for pituitary hormones. Patients may present with pituitary hypofunction, visual disturbances, headache, nausea and vomiting. The clinical course is usually benign. 